\title{
CRESCIMENTO DE NANOPARTÍCULAS DE PRATA: INFLUÊNCIA DO ESTABILIZANTE
}

\author{
Claudio H. Rodrigues ${ }^{1 *}$, Adriana Fontes ${ }^{2}$, Juliana S.A. Falcão ${ }^{3}$, Goreti Pereira ${ }^{4}$, Beate Santos ${ }^{1}$ \\ ${ }^{1}$ Departamento de Ciências Farmacêuticas, UFPE \\ ${ }^{2}$ Departamento de Biofísica e Radiobiologia, UFPE \\ ${ }^{3}$ Curso de Farmácia, Centro de Educação e Saúde, Universidade Federal de Campina Grande \\ ${ }^{4}$ Departamento de Química Fundamental, UFPE \\ *claudiohrodrigues@live.com
}

\begin{abstract}
INTRODUÇÃO
As nanopartículas de Prata (AgNPs) são sistemas coloidais (5 - 100 $\mathrm{nm}$ ) formados através dos fenômenos de nucleação e crescimento, por meio de um precursor, sendo o nitrato de prata o mais utilizado. Sua utilização é ampla, com foco em plasmônica, desenvolvimento de sensores e dispositivos ópticos, além de sua atividade antimicrobiana (BAPTISTA, 2012) e também na terapia fotodinâ mica (MELO, 2012).

A forma das nanoestruturas de prata pode ser controlada através das condições experimentais utilizadas. Os núcleos podem ser formados previamente, sendo denominados sementes e caracterizadas por dímeros de prata $(\mathrm{Ag}-\mathrm{Ag}) \mathrm{n}$, e o crescimento é promovido através da adição de volumes distintos destes numa solução do precursor de parta, ocorrendo nucleação e crescimento em etapas distintas da síntese, como descrito na metodologia de Aherne (2008). Em uma síntese, um dos destinos do precursor é aglomeração ao ponto de formar pequenas partículas, que tem a possibilidade de continuar crescendo em solução, devido ao movimento browniano desse mesmo sistema, e a velocidade dos processos da síntese pode variar substancialmente dependendo da técnica empregada (SAADE, 2013).
\end{abstract}

$O$ agente redutor utilizado pode determinar a velocidade dos processos. Um agente mais fraco promove o crescimento em um tempo mais lento, enquanto a adição de um agente forte acelera esse processo (SAADE, 2013). Segundo Aherne (2008), a cinética da reação para a obtenção das AgNPs a partir das sementes préformadas não ocorre de forma reprodutível, podendo-se obter cristais com forma e tamanhos indefinidos, pois sementes com diferentes estruturas crescem em solução, e consequentemente, originam morfologias distintas ao final do procedimento.

Afim de se promover o controle da forma e tamanho final das partí culas, são utilizados agentes surfactantes na produção da semente, que são caracterizados por polímeros com ou sem carga, e pode promover estabilidade ao sistema ou relacionar-se diretamente com o a formação do material, sendo um fator de fundamental importância na síntese. Aqueles que possuem carga, como o Poliestireno Sulfonato de Sódio (PSSS) influencia diretamente na deformação do cristal (AHERNE, 2008), enquanto polímeros sem carga como a Polivinilpirrolidona (PVP) confere estabilidade ao sistema, através de um sistema de malha auto organizada (MELO $J R, 2012$ ), promovendo também defeitos no cristal, formando nanoprismas (METRÁUX, 2005).

Este estudo teve como objetivo a síntese de AgNPs utilizando diferentes polímeros, com ou sem carga, relacionando parâmetros da síntese e resultados macroscópicos do sistema (turbidez, coloração), assim como a análise da absorção e do crescimento, por meio do deslocamento da banda.

\section{MATERIAIS E MÉTODOS}

Os sistemas foram preparados seguindo duas etapas: produção de sementes e crescimento posterior delas.

Preparação da Semente:

Em um experimento típico, sob temperatura ambiente e agitação à $1100 \mathrm{rpm}$, sementes de prata foram produzidas em meio aquoso combinando-se solução de citrato de sódio $\left(5 \mathrm{~mL}, 2,5 \mathrm{mmol} . \mathrm{L}^{-1}\right)$, solução do polímero desejado (PSSS), quitosana peso molecular mé dio, PVA, alginato de sódio ou polifosfato de sódio, obtidos da Sigma Aldrich e carbopol $940^{\circledast}$ obtido da Anastácio $(0,25 \mathrm{~mL}, 500$ $\left.\mathrm{mg} \cdot \mathrm{L}^{-1}\right)$ e solução de borohidreto de sódio $\left(\mathrm{NaBH}_{4}(0,3 \mathrm{~mL}, 10\right.$ mmol. $L^{-1}$, recém preparada), seguido pela adição de solução aquosa de $\mathrm{AgNO}_{3}$ ( $5 \mathrm{~mL}, 0,5 \mathrm{mmol} . \mathrm{L}^{-1}$, gota a gota). A amostra foi armazenada por dois dias antes da etapa do crescimento, para estabilização do coloide.

\section{Crescimento das partículas:}

Utilizando as mesmas condições da produção da semente, combinou-se $5 \mathrm{~mL} \mathrm{H}_{2} \mathrm{O}$, ácido ascórbico ( $75 \mathrm{~mL}, 10 \mathrm{mmol}^{-1}{ }^{-1}$ ) e volumes variados da semente $(40,60,90,120,200,260,400,500$ e $650 \mu \mathrm{L})$ previamente preparada, seguido pela adição de solução aquosa de $\mathrm{AgNO}_{3}\left(3 \mathrm{~mL}, 0,5 \mathrm{mmol} . \mathrm{L}^{-1}\right)$. A amostra foi armazenada por dois dias, para estabilização do coloide, antes de ser realizada a sua análise.

1.1 Espectroscopia UV-Vis

Os sistemas coloidais foram caracterizados por espectroscopia de absorção UV-Vis (espectrofotômetro modelo Lambda 650 UV/Vis, fabricante Perkin Elmer), na região de 350 a $800 \mathrm{~nm}$. Foram observados o crescimento da banda e a diminuição da absorbâ ncia, que representa o consumo da semente.

\section{RESULTADOS E DISCUSSÃO}

Nos espectros de absorção da semente, mostrados na Figura 1, observa-se um padrão entre todas as sínteses realizadas, com exceção das realizados na presença do alginato e quitosana. 0 tamanho esperado para essas partículas é em torno de $20 \mathrm{~nm}$, apresentando absorção com máximo em $\lambda=400 \mathrm{~nm}$ (SAADE, 2013). 0 resultado sugere que não há interação forte com $0 \mathrm{Ag}^{+}$na presença da quitosana, pois o padrão espectral sugere que os nú cleos continuam a crescer resultando na banda de absorção larga, caracterizando grandes dispersos ou formação de aglomerados. De fato para estes sistemas foi observada um sistema turvo, sem uma coloração pronunciada como os outros sistemas coloidais contendo sementes. Com o alginato, tem-se a coloração similar às outras 
sementes, porém o espectro um pouco mais alargado evidencia alguma interferência na formação das sementes, provavelmente prolongando o tempo de crescimento, resultando num tamanho maior $\mathrm{d} \sim 30-40 \mathrm{~nm}$ (SAADE, 2013).

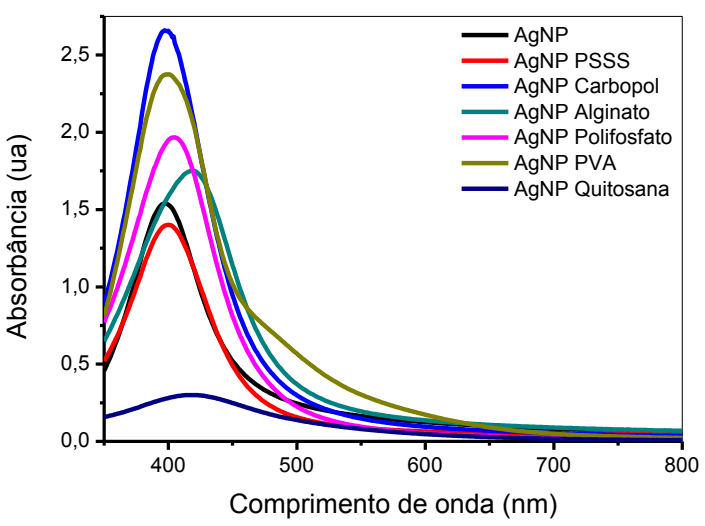

Figura 1 - Espectros de absorção dos sistemas coloidais contendo sementes de $\operatorname{Ag}(\mathbf{s})$, preparadas na presença de diferentes polímeros.

De uma forma geral, após o crescimento das partículas podemos constatar, observando-se os respectivos espectros de absorção eletrônica a presença de duas bandas: uma que evidencia a presença das sementes no meio (com máximos entre 400 - 430 $\mathrm{nm}$ ), e outra que refere-se as partículas que cresceram ou aglomeraram no sistema coloidal (com máximos entre 450 - 750 $\mathrm{nm})$. Uma concentração maior de sementes resulta em maiores quantidades de núcleos, e com omesmo volume da suspensão mãe contendo as sementes de $\mathrm{Ag}$ adicionado, observa-se núcleos menores, ocorrendo o contrário para uma menor concentração de sementes.

Esse padrão é observado independente do polímero utilizado, exceto para as partículas preparadas na presença da quitosana, onde foi observada aglomeração em todas as condições. Na síntese sem polímeros, foi observada aglomeração para partículas com volume menor de semente inicial (Figura 3), enquanto que para um volume maior de semente (Figura 2), observou-se uma maior estabilidade das suspenões, que pode estar relacionada com o efeito estabilizante promovido pelo citrato de sódio de meio, visto que não há um polímero para influenciar no crescimento das partí culas.

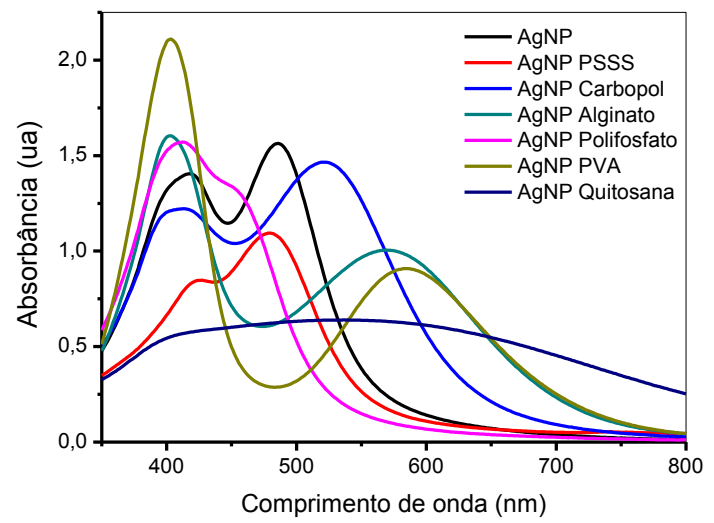

Figura 2 - Crescimento das partículas de Ag a partir da adição de $650 \mu \mathrm{L}$ da suspensão de sementes.

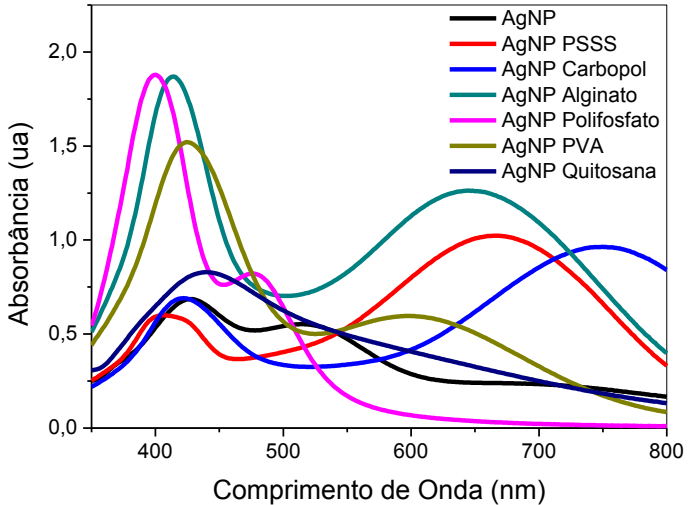

Figura 3 - Crescimento das partículas de Ag a partir da adição de $40 \mu \mathrm{L}$ da suspensão de sementes.

Não houve relação entre a carga do polímero e o padrão de crescimento das partículas. No meio contendo PVA, observou-se uma maior estabilização das sementes, que é observado como propriedade para polímeros neutros (MELO JR, 2012), ocorrendo então mínimo consumo desse sistema, enquanto em polímeros com carga negativa como o Polifosfato, o crescimento não foi acentuado, sendo originado um perfil bimodal de partículas.

Em contraste, as partículas submetidas à presença de carbopol, PSSS e alginato cresceram de forma extensiva e mantiveram um perfil espectral semelhante, sugerindo uma morfologia também semelhante, neste caso, o de discos prismáticos (AHERNE et al., 2008). O resultado foi demonstrado para o PSSS, por Aherne e colaboradores (2008), e é decorrente da preferência do polímero por uma das faces cristalinas das sementes de $\mathrm{Ag}$, e induzindo a anisotropia em crescimento posterior.

\section{CONCLUSÕES}

Conclui-se que as AgNPs na presença de diferentes polímeros surfactantes, demonstram diferentes modelos de crescimento, que podem ser associados às interações moleculares/carga com a superfície das sementes. As AgNPs crescidas na presença dos polí meros carbopol e alginato mostraram cinética reacional semelhantes à observada para o PSSS, sugerindo o uso destes agentes poliméricos biocompatíveis para a estabilização de nanopartículas de Ag para futuras aplicações, principalmente, biomédicas e fotônicas.

\section{REFERÊNCIAS}

AHERNE, D.; LEDWITH, D. M.; GARA, M.; KELLY, J. M. Optical Properties and Growth Aspects of Silver Nanoprisms Produced by a Highly Reproducible and Rapid Synthesis at Room

Temperature. Advanced Functional Materials. v. 18, p 2005-2016, 2008.

BAPTISTA, A.; Kato, I. T.; Prates, R. A.; Suzuki, L. C.; Raele, M. P.; Freitas, A. Z.; Ribeiro, M. S. Antimicrobial photodynamic therapy as a strategy to arrest enamel demineralization: a short-term study on incipient caries in a rat model. Photochemistry and Photobiology, v. 88, p. 584-589, 2012.

MELO, L. S. A. Síntese de nanoparticulas metálicas para aplicação em terapia fotodinâmica e espectroscopia. 153f. Dissertação (Doutorado), Centro De Ciências Exatas e da Natureza, Universidade Federal de Pernambuco, Recife, 2011.

MELO JR et al. Preparação de nanopartículas de prata e ouro: um método simples para a introdução da nanociência em laboratório de ensino. Química Nova. v.35, no.9, 2012.

METRAÚX, G.S., MIRKIN, C.A. Rapid thermal synthesis of silver nanoprisms with chemically tailorable thickness. Advanced Materials v. 17, p. 412-415, 2005. 
SAADE, J. Síntese/Fabricação e caracterização de micro e nanoestruturas para aplicação na fotônica e plasmônica. $134 f$.

Dissertação (Doutorado), Centro De Ciências Exatas e da Natureza,

Universidade Federal de Pernambuco, Recife, 2013 